\title{
Assessment of maternal referral systems used for a rural Zambian hospital: the development of setting specific protocols for the identification of complications
}

\author{
Ashley E Benson, Michael J Benson, Amy H Luke
}

Loyola University Stritch School of Medicine, Center for Community and Global Health.

Emails: Amy Luke: aluke@luc.edu, Michael Benson: michael.benson@hsc.utah.edu, Ashley Benson: ashley.e.benson@hsc.utah.edu

\begin{abstract}
Background: In resource-limited countries, it is estimated that up to $75 \%$ of maternal deaths are preventable. Maternal referral systems are an effective measure to help prevent these deaths.

Objective: The objective of this study was to delineate criteria that health care workers use to identify obstetrical emergencies and make referrals, in order to evaluate the effectiveness of the established referral system and to implement improvements to this system.

Methods: Using a qualitative study design, the individuals with the highest level of formal obstetrics training at 10 health posts that refer to a rural Zambian hospital were surveyed using semi-structured interviews regarding their referral protocols. Data were analyzed through open-coding. At the conclusion of the interview, standardized referral protocols for obstetric emergencies derived from published guidelines and local practices were distributed.

Results: Identified complications resulting in referral most commonly included post-partum hemorrhage $(70 \%)$, prolonged labor $(70 \%)$, malpresentation (50\%), antepartum hemorrhage (40\%), and retained placenta (40\%). While numerous reasons for referral were identified, there was little consensus on the referral protocol used for each complication. Obstacles to successful referral most commonly included cellular network disruptions (70\%), distance (50\%), and lack of transportation $(30 \%)$.

The referral protocols distributed to health posts covered only 11 of the 23 complications cited as the most common reason for referral.

Conclusion: The referral criteria and protocols were updated to include all of the reported complications. We propose this document for others working in resource-limited settings attempting to establish or evaluate a maternal referral systems.

Keywords: Maternal referral systems, Zambian hospital, protocols, complications.

DOI: https://dx.doi.org/10.4314/ahs.v19i1.27

Cite as: Benson AE, Benson MJ, Luke AH. Assessment of maternal referral systems used for a rural Zambian hospital: the development of setting specific protocols for the identification of complications. Afri Health Sci. 2019;19(1). 1536-1543. bttps:// dx.doi.org/10.4314/abs.v19i1.27
\end{abstract}

\author{
Corresponding author: \\ Michael J Benson, \\ Department Address: \\ University of Utah, Department of Anesthesiology \\ 30 N 1900 E, RM 3C444 SOM \\ Salt Lake City, Utah 84132 \\ Department Phone and Fax: \\ Phone: 801-581-6393; Fax: 801-581-4367 \\ Phone: 801-833-6500 \\ Email: michael.benson@hsc.utah.edu
}

\section{Introduction}

Maternal mortality ratios are powerful expressions of health systems outcomes, and to some extent, gender discrimination throughout the world.1,2 The United Nation's Sustainable Development Goals (SDG) resolve to reduce the maternal mortality to less than 70 per 100,000 live births, ${ }^{3}$ however, despite numerous attempts by the World Health Organization (WHO), the United Nations Children's Fund (UNICEF), and the United Nations Population Fund (UNFPA) there has been little improvement. ${ }^{4}$ Of the direct contributors to maternal mortality,

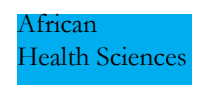

(C) 2019 Benson et al. Licensee African Health Sciences. This is an Open Access article distributed under the terms of the Creative commons Attribution License (https://creativecommons.org/licenses/BY/4.0), which permits unrestricted use, distribution, and reproduction in any medium, provided the original work is properly cited. 
the most common include sepsis, eclampsia, obstructed labor, unsafe abortion, and hemorrhage. ${ }^{5}$ It is estimated that $75-90 \%$ of maternal mortalities are preventable in resource-limited settings, ${ }^{6-8}$ and that essential obstetric care for hemorrhage, sepsis, eclampsia, and obstructed labor alone could prevent half of these. ${ }^{8}$ There is extensive evidence that obstetric emergencies managed in hospitals encounter reduced maternal mortality, ${ }^{9}$ and that providers require protocols to assist in determining at which point a woman requires referral to higher level care. ${ }^{10}$ Various models explain gaps in referral systems, such as the ' 3 Delays Model $^{\prime 11}$, which which cites delays in identifying and reaching appropriate facilities as one of the main factors affecting timely care of pregnant women and thus contributing to complications. Despite this, current data suggests that the number of complicated deliveries at referral centers falls well below the estimated need ${ }^{12}$, and that "maternity referral systems are under-documented, under-researched, and under-theorized". ${ }^{13}$

Zambia is a land-locked sub-Saharan country sharing borders with eight surrounding countries, with an estimated population of fourteen million people. ${ }^{14}$ The rate of maternal morality in Zambia is 591 per 100,000 live births, ${ }^{15}$ corresponding to a lifetime risk of maternal death of 1 in 27 women. ${ }^{16}$ While the top five most common direct causes of maternal death in Zambia correspond to the aforementioned, sepsis is associated with nearly $50 \%$ of Zambian maternal deaths. ${ }^{8}$ In Zambia, limited human resources exacerbate an already large healthcare burden, with the government only able to employ $40 \%$ of the clinicians required to staff health facilities, and rurally, individuals, who have no clinical training, staff some health posts. Consequently, in rural Zambia, only $31 \%$ of deliveries are assisted by a skilled health worker. ${ }^{15}$ The maternal referral system in Zambia is from the community to health posts and subsequently to district, general, and central hospitals. This system relies on the patient's ability to recognize a complication and seek care, and subsequently the health worker's ability to recognize and take timely action on any complication that may arise. ${ }^{17}$ Murray et $\mathrm{al}^{13}$ identified a need for "setting specific protocols" in effective maternal referral systems.

Our study was in collaboration with the Lumezi Mission hospital, which operates as a level one mission-hospital in the Eastern Province of Zambia. It currently has one vol- unteer doctor on staff, but functions as a comprehensive provider of emergency obstetric services. ${ }^{18}$ Its catchment area covers 1000 square kilometers, covering an estimated population of 70,000 people, ${ }^{19}$ and receives up to 500 ambulatory patients daily. ${ }^{20}$ At time of the study, there were no specific maternal referral policies or protocols implemented in Lumezi, however the district has recently adopted widespread use of the WHO partograph. To identify criteria that health posts use to refer maternal complications to higher-level care and to standardize referral protocols, we surveyed all health posts that refer to a rural level one district hospital in Lumezi, Zambia. In doing so, our aims were to qualitatively identify experienced complications and how the referral of patients was managed. Following the survey, health care workers were provided standardized, setting specific protocols.

\section{Methods \\ Study design}

A cross sectional descriptive study was conducted using qualitative methods. We conducted semi-structured interviews at the ten health posts that refer to the Lumezi Mission-Hospital. Interview administered surveys addressed the following domains: perceived causes of maternal morality; how these causes are recognized; for what conditions are mothers referred to higher-level care, for each identified cause whether a referral protocol exists and the criteria used; and what obstacles interfere with effective referrals. Following the survey, health care workers were provided standardized, setting specific protocols.

\section{Sample size and sampling technique}

All of the ten health posts that refer to Lumezi Mission-Hospital were included in the study. These included: Chanyalubwe, Chickomeni, Mwasempangwe, Mwasempangwe 35, Mwimba, Ndiwala, Ng'onga, Nkhanyu, Umi, and Zumwanda.

\section{Data collection}

Data collection was carried out over a four-week period between June and July 2013. The interview was unscheduled and at each health post, the skilled health worker with the most formal training in obstetrics was requested to complete a survey. The surveys were interview-administered in English; and a Lumezi Mission-Hospital volunteer assisted with translation as necessary. The authors recorded the responses. 


\section{Ethical considerations}

Ethical clearance was obtained from the Institutional Review Board (IRB) prior to data collection. A letter of support was obtained from Lumezi Mission-Hospital. Participation was voluntary, and participants had the opportunity to ask questions, decline participation, or withdraw from the study at any time. Interviews were conducted in locations that ensured privacy with minimal interruptions. No information regarding the health workers' knowledge was shared with their supervisors or colleagues. Data was recorded on de-identified forms and was securely handled by the authors using password protection on the computer. After all data was entered in the computer, the questionnaires were stored securely.

\section{Data analysis}

All questionnaires were de-identified following administration. All data was pooled, coded, and assessed as a whole.

\section{Results}

Study personnel visited all ten of the health posts referring to a Level One Hospital in Lumezi, Zambia. Table 1 represents the position of the health care worker surveyed at each site. At the time of visit, $30 \%$ of the referral clinics had personnel formally trained in obstetrics present. One clinic had no one formally trained in obstetrics on staff.

Table 1. Type of health post worker surveyed at each site.

\begin{tabular}{ll}
\hline Health Post Worker Surveyed & Percentage of total \\
\hline Community Health Assistant & $30 \%$ \\
Classified Daily Employee & $30 \%$ \\
Environmental Health Technologist & $10 \%$ \\
Nurse & $10 \%{ }^{\mathrm{a}}$ \\
Clinical Officer & $10 \% 0^{\mathrm{a}}$ \\
Midwife & $10 \% 0^{\mathrm{a}}$ \\
\hline
\end{tabular}

a indicates individuals formally trained in obstetrics.

Health post workers were asked to identify most common complications leading to maternal referral and describe any associated protocols used in the referral process. Table 2 shows that the most commonly cited reasons for maternal referral included post-partum hemorrhage (70\%), prolonged labor $(70 \%)$, malpresentation (50\%), antepartum hemorrhage $(40 \%)$, and retained placenta (40\%); some health posts named multiple complications as the most common. While numerous reasons for referral were identified, there was little standardization of the protocols for referral for each complication. Of these most common reasons for referral cited by health post workers, only 11 of the 23 complications were included in the original referral criteria and protocols' document provided to the health post workers.

Table 2 further demonstrates that there was poor overlap between health post referral criteria and the proposed referral criteria. For each complication, the responses matched the standardized referral protocols as follows: ante-partum hemorrhage $(40 \%)$, post-partum hemorrhage $(40 \%)$, prolonged labor $(30 \%)$, malpresentation $(20 \%)$, retained placenta $(20 \%)$, obstructed labor $(10 \%)$, eclampsia $(10 \%)$, young maternal age $(10 \%)$, previous c-section $(0 \%)$, fetal distress $(0 \%)$, and cephalopelvic disproportion $(0 \%)$. 
Table 2. Major causes for referral offered by health post workers.

\begin{tabular}{|c|c|c|c|}
\hline Complication & $\begin{array}{l}\text { Percentage of } \\
\text { health posts } \\
\text { naming } \\
\text { complication }\end{array}$ & $\begin{array}{l}\text { Complication } \\
\text { covered in } \\
\text { Referral Criteria } \\
\text { and Protocols } \\
\text { Document }\end{array}$ & $\begin{array}{l}\text { Proportion of } \\
\text { referral criteria } \\
\text { consistent with } \\
\text { proposed } \\
\text { referral } \\
\text { guidelines } \\
\end{array}$ \\
\hline Postpartum hemorrhage & $70 \%$ & Yes & $40 \%$ \\
\hline Prolonged Labor & $70 \%$ & Yes & $30 \%$ \\
\hline Malpresentation & $50 \%$ & Yes & $20 \%$ \\
\hline Antepartum hemorrhage & $40 \%$ & Yes & $40 \%$ \\
\hline Retained placenta & $40 \%$ & Yes & $20 \%$ \\
\hline Obstructed Labor & $30 \%$ & Yes & $10 \%$ \\
\hline Small motherb & $30 \%$ & No & -- \\
\hline Previous C-section & $30 \%$ & Yes & $0 \%$ \\
\hline Fetal Distress & $20 \%$ & Yes & $0 \%$ \\
\hline Anemiab & $20 \%$ & No & -- \\
\hline Young maternal age & $20 \%$ & Yes & $10 \%$ \\
\hline HIVb & $20 \%$ & No & -- \\
\hline Cord Prolapse $^{b}$ & $10 \%$ & No & -- \\
\hline Pre-eclampsiab & $10 \%$ & No & -- \\
\hline Eclampsia & $10 \%$ & Yes & $10 \%$ \\
\hline $\begin{array}{ll}\text { Pelvic } & \text { Inflammatory } \\
\text { Disease }^{b} & \end{array}$ & $10 \%$ & No & -- \\
\hline Convulsions $^{\mathrm{b}}$ & $10 \%$ & No & -- \\
\hline Edemab $^{\mathrm{b}}$ & $10 \%$ & No & -- \\
\hline Primiparous ${ }^{b}$ & $10 \%$ & No & -- \\
\hline Spontaneous abortions ${ }^{b}$ & $10 \%$ & No & -- \\
\hline Cephalopevlic Disproportion & $10 \%$ & Yes & $0 \%$ \\
\hline $\begin{array}{l}\text { Delayed presentation to } \\
\text { hospital/home delivery }^{b}\end{array}$ & $10 \%$ & No & -- \\
\hline Placenta previab & $10 \%$ & No & -- \\
\hline
\end{tabular}

Table 3 indicates the responses of health post workers when asked directly about the performance of specific screening criteria. $100 \%$ reported assessment of HIV status, fetal position, and post-partum blood pressure. $90 \%$ of health posts reported monitoring of antenatal blood pressure, post-partum temperature, and post-partum heart rate. The least used screening criteria were pelvic size assessment (40\%), urine protein levels (30\%), and post-partum hemoglobin (10\%). 
Table 3. Screening criteria for antenatal and post-partum mothers.

\begin{tabular}{|c|c|}
\hline Screening Criteria & Rate \\
\hline HIV Screening & $100 \%$ \\
\hline Fetal Position & $100 \%$ \\
\hline $\begin{array}{l}\text { Post-partum: Blood Pressure } \\
\text { Check }\end{array}$ & $100 \%$ \\
\hline $\begin{array}{l}\text { Antenatal Blood Pressure } \\
\text { Check }\end{array}$ & $90 \%$ \\
\hline Post-partum: Temperature & $90 \%$ \\
\hline Post-partum Heart Rate & $90 \%$ \\
\hline $\begin{array}{l}\text { Post-partum:Estimated Blood } \\
\text { Loss }\end{array}$ & $80 \%$ \\
\hline Post-partum Respiratory Rate & $50 \%$ \\
\hline Pelvic Size Assessment & $40 \%$ \\
\hline Urine Protein Levels & $30 \%$ \\
\hline Post-partum: Hemoglobin & $10 \%$ \\
\hline
\end{tabular}

Health post workers noted numerous challenges in the referral process with the most prevalent being an inability to communicate due to a poor cellular network $(70 \%)$ and distance from the referral hospital (50\%). Lack of a vehicle by the referral hospital (30\%) and lack of use of the radio system by the referral hospital $(30 \%)$ were also commonly cited. Other less common challenges included lack of feedback after referrals $(20 \%)$, and a lack of trained staff at the health post $(10 \%)$. (Table 4$)$

Table 4. Perceived challenges in the referral process.

\begin{tabular}{ll}
\hline Challenges in Referral Process & Percentage Responding \\
\hline Poor Cellular Network & $70 \%$ \\
Distance & $50 \%$ \\
Hospital does not use radio system & $30 \%$ \\
No vehicle available at referral hospital & $30 \%$ \\
Lack of feedback from referral & $20 \%$ \\
Lack of trained staff at post & $10 \%$ \\
\hline
\end{tabular}

\section{Discussion}

The SDG resolves to reduce maternal mortality by $2030 .^{3}$ The WHO, UNICEF, and UNFPA have all indicated a need for improved access to emergency obstetric care as the best mechanism for decreasing maternal mortality in low-income countries. ${ }^{9} 18$ It has been theorized that approximately $15 \%$ of pregnancies with complications require modern obstetric care, ${ }^{21}$ however current data suggests that referral systems are far from sufficient and the number of complicated deliveries handled at higher-level care centers falls well below this estimated need. ${ }^{12}$ Furthermore, it is accepted that providers require pro- 
tocols for determining when referral to higher-level care is needed and to reduce unnecessary delay. ${ }^{10,22}$ To delineate criteria that health posts use to refer to higher-level care and to standardize referral protocols, we surveyed all health posts that refer to a rural level one district hospital in Lumezi, Zambia. Following the survey, healthcare workers were provided standardized, setting specific protocols. We found that upon arrival at the health posts, few had skilled health workers present; there was little standardization of referral criteria for a given complication; cellular network disturbances, transportation, and lack of feedback were common obstacles to successful referral; and that our proposed referral protocols only broached 11 of the 23 common referral causes elucidated in interviews. One limitation of this study is the small participant pool (although it includes all health posts in the given catchment area) and that the data collection relies on perceived opinions of the respondents. Ability to review more objective tools such as case audits would have helped greatly; however, these were unavailable at time of study.

Upon arrival to the health posts, an unexpected finding was that although $90 \%$ of the centers had a skilled health worker on staff, skilled health workers were only present at three of the ten centers. There were various explanations for their absences, including being on indefinite leave, taking a short break, or attendance at conferences. The staff shortages that affect low-income countries have been well documented, ${ }^{6}$ however, these results demonstrate that even in the presence of adequate staff placements, the systems can tolerate very few unanticipated absences.

Importantly, we found that there was poor overlap between referral criteria used by each health post and the proposed referral criteria. More so, the referral criteria delineated varied considerably from one health post to another. For example, the criteria given by health posts for post-partum hemorrhage referral ranged from: "after one hour", "500 milliliter blood loss", "after three hours", or "immediately, if tears have been sutured". Certainly, one of the limitations of this study and potential explanations for the poor overlap in these responses is the varied educational backgrounds of each health care worker interviewed. However, these responses reflect the criteria, or lack thereof, that is being used to assess obstetrical emergencies on a daily basis in this district. As referral of patients from basic to higher-level care providers is essential to the functionality of health systems ${ }^{23}$, this data demonstrates a need for standardization of referral criteria. While we distributed a set of proposed referral criteria, our document only covered 11 of the 23 complications for which the interviewed health care workers said they referred. This data further supports previous assertions that the planning of a referral system requires an understanding of the needs of the population, the capability of the health systems, and resources to meet those needs. ${ }^{13}$ And further, that setting-specific protocols should reflect local conditions, organizational capacity, and community preferences. 24 Our proposed protocols have been updated to reflect the community needs, and we propose these protocols as a starting point for those working in resource-limited settings. Although, much work has gone into referral systems at the national and international level, the district-level needs have been somewhat ignored, ${ }^{25}$ and so we recognize the need for these protocols to be adapted as necessary to meet the specific needs of various communities.

Again, maternal referral systems exist to identify the 15\% of pregnancies with complications require modern obstetric care. ${ }^{21}$ We propose the use of simple vital signs and physical exam findings that are used in the diagnosis of the major complications that contribute to maternal mortality (Table 3). Overall, health post workers affirmed that they use many of these screening criteria. Of those that were not frequently utilized (pelvic size assessment, urine protein levels, and hemoglobin levels), the majority of sites did not have the technology to carry out these assessments (particularly urine protein and hemoglobin levels). Interestingly, although obstructed labor accounts for $7 \%$ of maternal mortality in Zambia, ${ }^{15}$ only $40 \%$ of health posts responded that they assess pelvic size, although this is an inexpensive assessment.

The most common obstacles to successful referral were cellular network disturbances and inconsistent radio use and access to the higher-level care facility (either secondary to distance or availability of a vehicle). These issues 
of infrastructure are commonly encountered in low-resource settings, ${ }^{26}$ and previous studies have indicated that up to $73 \%$ of Zambian preventable deaths has systems factors identified as contributing factors. ${ }^{8}$ Interestingly, two of the ten health posts reported a lack of feedback following their referrals. This feedback is essential to the continued education of community health workers and also reinforcement when a health worker is questioning whether or not to make a referral. This finding that recommendations often focus on protocols for the referred, but protocols at the receiving facility may also require attention has been previously highlighted. ${ }^{13}$

\section{Conclusion}

The data that we have reported in this paper help to establish a baseline for the development of an effective maternal referral system in the district of Lumezi, Zambia and have been used to provide local heath posts with standardized referral criteria. In addition, we identified obstacles to successful referral that can be targeted in the future.

\section{Acknowledgments}

We would like to acknowledge and thank the entire staff of Lumezi Mission Hospital for allowing us work with them, Dr. Laura Heman, M.D., for her tireless help with the project and protocols document, Dr. Michael Breen, M.D., from Monze Mission Hospital, for the use of his protocols document.

\section{Conflict of interest}

None.

\section{References}

1. Thompson A. Poor and Pregnant in Africa: safe motherhood and human rights. Midwifery, 1999(15): p. 146153.

2. WHO, Gender and Health: Technical Paper, WHO/ FRH/WHD/98.16, Editor. 1998, WHO: Geneva.

3. Transforming our world : the 2030 Agenda for Sustainable Development. 2015, UN General Assembly.

4. Starrs, A.M., Safe motherhood initiative: 20 years and counting. Lancet, 2006. 368(9542): p. 1130-2.

5. Ghosh MK. Maternal mortality. A global perspective. J Reprod Med, 2001. 46(5): p. 427-33.

6. Fawcus $\mathrm{S}$, et al. A community-based investigation of avoidable factors for maternal mortality in Zimbabwe. Stud Fam Plann, 1996. 27(6): p. 319-27.

7. Wagstaff A, M Claeson. The Millenium Development Goals for Health: Rising to the Challenges. 2004, The World Bank: Washington DC.

8. Kilpatrick SJ et al. Preventability of maternal deaths: comparison between Zambian and American referral hospitals. Obstet Gynecol, 2002. 100(2): p. 321-6.

9. Van Lerberghe W, V De Brouwere, Of Blind Alleys and Things that Have Worked: History's Lessons on Reducing Maternal Mortality, in Safe Motherhood Strategies: a Review of the Evidence, V. De Brouwere and W. Van Lerberghe, Editors. 2001, ITG Press: Antwerp. p. 7-34. 10. Jahn A, V De Brouwere. Referral in Pregnancy and Childbirth: Concepts and Strategies, in Safe Motherhood Strategies: a Review of the Evidence, V. De Brouwere and W. Van Lerberghe, Editors. 2001, ITG Press: Antwerp. p. 225-242.

11. Thaddeus S, D Maine. Too far to walk: maternal mortality in context. Soc Sci Med, 1994. 38(8): p. 1091-110.

12. Jahn A et al. Maternity care in rural Nepal: a health service analysis. Trop Med Int Health, 2000. 5(9): p. 65765.

13. Murray SF, SC. Pearson. Maternity referral systems in developing countries: current knowledge and future research needs. Soc Sci Med, 2006. 62(9): p. 2205-15.

14. Zambia. World Factbook 2013 cited 201427 February 2014; Available from: https://www.cia.gov/library/ publications/the-world-factbook/geos/za.html.

15. Zambia Demographic and Health Survey 2007. 2009, Central Statistical Office (CSO), Ministry of Health $(\mathrm{MOH})$, Tropical Diseases Research Centre (TDRC), University of Zambia, and Macro International Inc.: Calverton, Maryland.

16. Maternal Mortality in 2005: Estimates developed by WHO, UNICEF, UNFPA, and The World Bank. 2007, World Health Organization: Geneva.

17. Alwar J, V Mtonga, B Sikatoye. Report of the Summative Evaluation of the Essential Obstetric Care Project in Mpongwe, Masaiti and Lufwanyama. 2000, UNICEF. 18. WHO, UNICEF, and UNFPA, Guidelines for Monitoring the Availability and Use of Obstetric Services. 1997: New York.

19. VSO. Lumezi Mission Hospital (Zambia). 2014; Available from: http://vso.force.com/jobopportunities/ Job_DetailsPage?jid=a0mD0000003P8BnIAK. 
20. Lumezi Hospital Foundation. 2013 cited 201426 February; Available from: http://www.lumezihospital. $\mathrm{com} /$.

21. Adamson P. A failure of Imagination, in Progress of the Nations. 1996, UNICEF.

22. Maine D. Safe Motherhood Programs: Options and Issues. 1991, New York: Center for Population and Family Health.

23. WHO and UNICEF, Primary Health Care, in International Conference on Primary Health Care. 1978: Geneva.
24. Sauerborn R et al. Assessment of MCH services in the district of Solenzo, Burkina Faso. III. Effectiveness of $\mathrm{MCH}$ services in detecting of and caring for mothers and children at risk. J Trop Pediatr, 1989. 35 Suppl 1: p. 14-7.

25. WHO, Selecting Reproductive Health Indicators: A guide for district managers, WHO/RHT/HRP/97.25, Editor. 1997: Geneva.

26. Krasovec K. Auxiliary technologies related to transport and communication for obstetric emergencies. Int J Gynaecol Obstet, 2004. 85 Suppl 1: p. S14-23. 